NBER WORKING PAPER SERIES

\title{
PRODUCTIVITY MEASUREMENT WITH NONSTATIC EXPECTATIONS AND VARYING CAPACITY UTILIZATION: AN INTEGRATED APPROACH
}

Catherine J. Morrison

Working Paper No. 1561

NATIONAL BUREAU OF ECONOMIC RESEARCH 1050 Massachusetts Avenue Cambridge, MA 02138 February 1985

Research was supported by the National Science Foundation under Grant No. SES-8309352 is gratefully acknowledged. The research reported here is part of the NBER's research program in Productivity. Any opinions expressed are those of the author and not those of the National Bureau of Economic Research. 


\author{
Productivity Measurement with \\ Nonstatic Expectations and Varying \\ Capacity Utilization: \\ An Integrated Approach
}

\section{ABS TRACT}

Typically measures of multifactor productivity growth have been based on a production and optimization framework that assumes all inputs are instantaneously adjustable, thus ignoring the important impacts of short run fixity of certain inputs. This paper focuses on the distinction between short and long run production behavior represented by economic capacity utilization indexes, and on the adjustment of observed productivity measures for the effects of short run fixity characterized by these indexes. A dynamic optimization model based on adjustment costs for quasi-fixed inputs is developed to calculate capacity utilization adjustments for productivity growth measures. The resulting framework is then used to identify empirically the effects of capacity utilization, nonstatic expectations, nonconstant returns to scale and adjustment costs for both capital and labor on productivity growth in the U.S. manufacturing sector, 1947-1979.

Catherine J. Morrison Nationa 1 Bureau of Economic Research 204 Junipero Serra Blvd. Stanford, CA 94305 


\section{INTRODUCTION}

Measures of multifactor productivity growth have long been linked to outward shifts in production isoquants or downward shifts in average cost curves. Typically, such links have been built on a production and optimization framework that assumes all inputs are instantaneously adjustable, and thus assumes also that there is no distinction between short and long run. This distinction can be very important, however, particularly when large shocks occur, for in such cases the characteristics of the firm's short run behavior during the initial part of the adjustment response may differ substantially from those occurring once long run equilibrium has been attained. This paper focuses attention on the implications for multifactor productivity measurement of increasing marginal adjustment costs for quasi-fixed inputs, and thus on the need to distinguish short- from long-run impacts.

Most of the models currently used for productivity analysis are based on the assumptions of full utilization or long run equilibrium and static expectations at all points in time for all inputs. More specifically, these models are typically based on the assumption that firms always use both technically and economically efficient input combinations. Thus productivity growth may be represented by $\partial \ln Y / \partial t-\varepsilon_{f t}$ (where $Y$ is output, defined by the production function $Y=f(x)$, and $t$ represents the state of technology), or by $\partial \operatorname{lnC} / \partial t-\varepsilon_{\mathrm{ct}}$ (where $\mathrm{C}$ is total costs). Ohta (1975) has developed the dual relationship between $\varepsilon_{f t}$ and $\varepsilon_{\text {ct }}$ by showing that with constant returns to scale (CRTS) $\varepsilon_{f t}=\varepsilon_{\text {ct }}$ and with nonconstant returns to scale (NCRTS) $\varepsilon_{\mathrm{ft}}=\varepsilon_{\mathrm{ct}} \varepsilon_{\mathrm{cy}}^{-1}$, where $\varepsilon_{\mathrm{cy}}=\partial \ln \mathrm{C} / \partial \ln Y$. 
These representations of productivity, however, permit no distinction between short and long run since they are essentially based on a static or timeless model where all inputs are assumed instantaneously variable.

This problem was recognized in early studies of technical change such as that by Jorgenson and Griliches (1967), who attempted to adjust productivity measures for cyclical adjustment using capacity utilization (CU) indices. Such cyclical adjustments, however, have ambiguous interpretation and have been a source of dispute since they are not based on a theoretical foundation within which the short run-long run nature of productivity growth is isolated.

More recently, Berndt and Fuss (1981) and Baily (1981) have attempted to capture the effect of "disequilibrium" using the deviation of market- and implicit-valuation of the fixed capital stock faced by the firm. These studies are of crucial importance for focussing attention on the impacts of input stocks. Ambiguities still remain, however, concerning the roles of dynamic optimization and anticipatory expectations.

In order to facilitate interpretation of utilization decisions and resulting implications for productivity growth, an integrating framework is required to capture variations in utilization, anticipatory behavior and costs of adjustment, as well as the effects of nonconstant returns to scale. Such a framework, a dynamic optimization model of an imperfectly competitive firm producing at a point of long run nonconstant returns to scale with increasing marginal adjustment costs on quasifixed inputs and nonstatic expectations, has been constructed by 
Morrison $(1983 \mathrm{~b}, 1984)$. The model is based on costs of adjustment for quasi-fixed inputs that induce slow adjustment by firms to "optimal" or "desired" levels of the quasi-fixed inputs consistent with nonstatic expectations on prices and output. The model is structured so one can derive explicitly a system of short run demand equations for variable inputs and accumulation equation(s) for quasi-fixed input(s) based on an endogenous "flexible accelerator" or partial adjustment process, as in Berndt, Fuss and Waverman (1979). Thus by characterizing the corresponding cost curves one can determine the firm's optimal adjustment path from the short to long run in response to a temporary disequilibrium situation.

In this paper I show how various impacts on observed productivity can be identified and measured within such a framework. The plan of the paper is as follows. In section II I first demonstrate that (i) multifactor productivity measures are affected systematically by divergence between long run equilibrium and short run (temporary) equilibrium, hereafter denoted subequilibrium, (ii) a properly defined index of capacity utilization (CU) can be used to quantify this divergence, and (iii) the existence of nonstatic expectations, adjustment costs, and nonconstant returns to scale affects the time path of investment and thus the time pattern of measured productivity growth through impacts on CU. In Section III I develop and report on an empirically implementable econometric model that permits separate identification of the effects of $\mathrm{CU}$, nonstatic expectations, adjustment costs and nonconstant returns to scale on productivity in the U.S. manufacturing sector, 1947-1979. A primary finding is that both 
subequilibrium and anticipatory behavior have substantial impacts on observed productivity measures. In addition, there is a notable difference between two alternative models used, which suggests that disaggregation of labor into production and nonproduction workers and recognition of the quasi-fixity of nonproduction workers are crucial for developing valid measures of productivity trends. Finally, in Section IV I focus on how this research integrates much of the previous literature on factor demands and productivity growth, and thus provides a framework within which to reconcile alternative approaches. 
II. PRODUCTIVITY AND ITS COMPONENTS: A THEORETICAL ANALYSIS

Productivity growth is defined as the diminution in total costs from an increase in the state of technology ( $t$ ) not explained by substitution between inputs--the residual $\varepsilon_{c t}$. In order empirically to identify the impacts on total productivity mentioned in the Introduction, a framework must be constructed in which deviations of capacity utilization (CU) from unity, nonstatic expectations, adjustment costs, and nonconstant returns to scaie (ÑCRTS) are incorporated. This requires recognizing the constraints on the firm which result in subequilibrium utilization of the quasi-fixed inputs. Thus the analysis is structured around a cost function divided explicitly into variable and fixed cost components. The output or price choice for a firm facing a demand curve with finite elasticity is assumed to be a sequential decision, taken into account in the estimating model. I begin by using this general framework to identify the current subequilibrium--CU--and technical progress components of observed productivity growth. Additional extensions are considered in turn.

Assume the restricted or variable cost function can be represented by $G\left(x_{j}, \dot{x}_{j}, w_{i}, t, Y\right)$ where $x_{j}$ is a vector of $J$, quasi-fixed input quantities, $\dot{x}_{j}$ is the time derivative of $x_{j}$, and $w_{i}$ is the vector of I variable input prices. Increasing marginal costs of adjustment due to $\dot{x}_{j} \neq 0$ affect variable costs according to $\partial G / \partial\left|\dot{x}_{j}\right| \geq 0$, $\partial^{2} G / \partial\left|\dot{x}_{j}\right|^{2} \geq 0$, and thus make the optimization problem a dynamic one. As outlined in Lau (1978) and Berndt, Fuss and Waverman (1979), G represents minimum variable costs given $x_{j}, w_{i}$ and $y_{\text {, and under }}$ appropriate regularity conditions has standard properties including: 


$$
\partial G / \partial w_{i}=v_{i}=\text { cost minimizing variable } i \text { input demand, and }
$$

b) $-\partial G / \partial x_{j}=z_{j}=$ shadow value for quasi-fixed input $j$.

A "shadow value of investment" can be defined analogously as:

c)

$$
-\partial G / \partial \dot{x}_{j}=z \cdot{ }_{j}=\text { the shadow value of } \dot{x}_{j}
$$

This expression represents the marginal cost savings involved if one less unit of investment is required to reach a given level of capital stock. It therefore represents the "flow" costs incorporated in the dynamic framework. ${ }^{1}$

Total costs are variable plus fixed costs or $C\left(Y, t, w_{i}, p_{j}, x_{j}, \dot{x}_{j}\right)=$ $G\left(Y, t, w_{i}, x_{j}, \dot{x}_{j}\right)+\sum_{j} p_{j} \cdot x_{j}$ where $p_{j}$ is a vector of market rental prices for the fixed inputs. The change in total costs from technical progress, represented by $d \ln C / d t$, is characterized by the full response of both variable and quasi-fixed inputs to long run equilibrium levels:

$$
\frac{d \ln C}{d t}=\frac{1}{C}\left[\left.\frac{d C}{d t}\right|_{x_{j}=\bar{x}_{j}}+\Sigma_{j} \frac{\partial C}{\partial x_{j}} \frac{d x_{j}}{d t}\right]
$$

This is a long run cost elasticity analogous to typical long run price and output elasticities. ${ }^{2}$ In effect the long run adjustment is a geometric series of adjustments toward the desired quasi-fixed input stocks $x^{*}$ which are assumed to close the "gap" between $x$ and $x^{*}$ by the proportion $\lambda$ in each period. This decomposition captures the flavor of the difference between short and long run producivity impacts, but is not very useful for interpretation or linkages with the notion of 
subequilibrium or $\mathrm{CU}$. To facilitate such an interpretation, for the moment assume CRTS and expand expression (2):

$$
\begin{aligned}
& \frac{d \ell n C}{d t}=\frac{1}{C}\left[\frac{\partial C}{\partial t}+\frac{\partial C}{\partial y} \frac{Y}{d t}+\Sigma_{i} \frac{\partial C}{\partial w_{i}} \frac{d w_{i}}{d t}+\Sigma_{j} \frac{\partial C}{\partial p_{j}} \frac{d p_{j}}{d t}\right. \\
& \left.+\sum_{j} \frac{\partial C}{\partial \dot{x}_{j}} \frac{d \dot{x}_{j}}{d t}\right]+\frac{1}{C}\left(\Sigma_{j} \frac{\partial C}{\partial x_{j}} \frac{d x_{j}}{d t}\right) \\
& =-\varepsilon_{C t}+\left(1-\Sigma_{j} \varepsilon_{C t}-\Sigma_{j} \varepsilon_{C j}\right) \frac{\dot{Y}}{Y}+\Sigma_{i} \frac{v_{\dot{i}} w_{i}}{C} \frac{\dot{w}_{i}}{w_{i}} \\
& +\sum_{j} \frac{p_{j} x_{j}}{c} \frac{\dot{p}_{j}}{p_{j}}+\sum_{j} \frac{\left(p_{j}-z_{j}\right) x_{j}}{C} \frac{\dot{x}_{j}}{x_{j}}-\sum_{j}-\frac{z \cdot \dot{x}_{j}}{C} \frac{\ddot{x}_{j}}{\dot{x}_{j}} .
\end{aligned}
$$

where $-\varepsilon_{C t}=\partial \ln C / \partial t, \quad \varepsilon_{C Y}=\partial \ln C / \partial \ln Y, \quad \varepsilon_{C j}=\partial \ln C / \partial \ln x_{j}=\left(p_{j}-z_{j}\right) x_{j} / C$, $\varepsilon_{\dot{C}_{j}}=\partial \ln C / \partial \ln \dot{x}_{j}=\left(-z \cdot \dot{j}_{j} / C\right)$, and $\left(1-\Sigma \varepsilon_{C j}-\Sigma \varepsilon_{\dot{C}_{j}}\right)=\varepsilon_{C Y}$ with constant returns to scale. ${ }^{3}$ Cost diminution is specified to be positive; $\varepsilon_{C t}=-\partial \ln C / \partial t$, so increasing productivity is represented by a larger instead of a smaller number. Note also that $\mathrm{dx}_{j} / \mathrm{dt}$ represents the effect of a change in $t$ on the desired long run capital stock and thus on investment.

$$
\text { Following Ohta (1975), note that since } c=\sum_{j} w_{i} v_{i}+\sum_{j} p_{j} x_{j} \text { full }
$$

long run $\dot{C} / C$ can also be represented as:

$$
\begin{aligned}
\frac{\dot{c}}{c} & =\Sigma_{i} \frac{\dot{w}_{i} v_{i}}{C}+\sum_{i} \frac{w_{i} \dot{v}_{i}}{C}+\sum_{j} \frac{\dot{p}_{j} x_{j}}{C}+\sum_{j} \frac{p_{j} \dot{x}_{j}}{C} \\
& =\Sigma_{i} \frac{v_{i} w_{i}}{C} \frac{\dot{w}_{i}}{w_{i}}+\sum_{i} \frac{w_{i} v_{i}}{C} \frac{\dot{v}_{i}}{v_{i}}+\sum_{j} \frac{x_{j} p_{j}}{C} \frac{\dot{p}_{j}}{p_{j}}+\sum_{j} \frac{p_{j} x_{j}}{C} \frac{\dot{x}_{j}}{x_{j}} .
\end{aligned}
$$


Setting these two expressions for $\dot{C} / C$ equal to each other and cancelling on both sides leaves:

$$
\begin{aligned}
& \Sigma_{i} \frac{w_{i} v_{i}}{C} \frac{\dot{v}_{i}}{v_{i}}=-\varepsilon_{C t}+\left(1-\Sigma \varepsilon_{C j}-\Sigma \varepsilon_{\dot{C}_{j}}\right) \frac{\dot{q}}{y}-\Sigma_{j} \frac{z_{j} x_{j}}{C} \frac{\dot{x}_{j}}{x_{j}} \\
& -\sum \frac{z_{j} \dot{x}_{j}}{C} \frac{\ddot{x}_{j}}{\dot{x}_{j}}, \text { or } \\
& \varepsilon_{C t}=\left(1-\Sigma \varepsilon_{C j}-\sum \varepsilon_{\dot{C}_{j}}\right) \frac{\dot{Y}}{y}-\Sigma_{i} \frac{{ }_{w_{i} v_{i}}}{C} \frac{\dot{v}_{i}}{v_{i}}-\Sigma_{j} \frac{z_{j} x_{j}}{C} \frac{\dot{x}_{j}}{x_{j}} \\
& -\sum_{j} \frac{Z_{j} \dot{x}_{j}}{C} \frac{\ddot{x}_{j}}{\dot{x}_{j}}
\end{aligned}
$$

This expression is in fact the conventional productivity residual calculated as the difference between the change in output and the actual share-weighted sum of the changes in inputs or, in the dual, as the change in costs less the actual share-weighted sum of the changes in input prices. Note that equation (5) is valid if long run equilibrium and CRTS exist. The expression appears different than is usually seen because of the explicit recognition of the fixed input constraint-$\varepsilon_{C_{j}} \neq 0$, and of non-zero investment in temporary equilibrium-- $\varepsilon_{\dot{C}_{j}} \neq 0$.

In the long run, however, (5) collapses to the usual expression since $z_{j}=p_{j}$ and $z_{j}=0$ so $\varepsilon_{C j}=\varepsilon_{\dot{C}_{j}}=0$ for all $j .4$ The importance of the representation of $\varepsilon_{\mathrm{Ct}}$ in (5) can be illuminated by dividing both sides by $\left(1-\Sigma_{i} \varepsilon_{C j}-\sum_{j} \varepsilon_{\dot{C} j}\right)$. This 
results in:

(6)

$$
\begin{aligned}
\frac{\varepsilon_{C t}}{1-\sum \varepsilon_{C j}-\sum \varepsilon_{C j}} & =\varepsilon_{C t}^{\prime}=\frac{\dot{Y}}{Y}-\frac{1}{1-\sum \varepsilon_{C j}-\sum \varepsilon_{C j} \dot{C}_{j}}\left[\sum_{i} \frac{w_{i} v_{i}}{C} \frac{\dot{v}_{i}}{v_{i}}\right. \\
& \left.+\Sigma_{j} \frac{z_{j} x_{j}}{C} \frac{\dot{x}_{j}}{x_{j}}+\Sigma_{j} \frac{z_{j} \dot{x}_{j}}{C} \frac{\ddot{x}_{j}}{\dot{x}_{j}}\right]
\end{aligned}
$$

Interpretation of this requires one more step. Note that

$$
\begin{aligned}
1-\Sigma \varepsilon_{C j}-\sum \varepsilon_{\dot{C} j} & =\frac{C-\Sigma{ }^{\left(p_{j}-z_{j}\right) x_{j}}+\Sigma z \cdot \dot{x}_{j}}{C}=\frac{G+\Sigma z_{j} x_{j}+\sum z \cdot \dot{x}_{j}}{C} \\
& =\frac{\text { shadow costs }}{\text { total costs }}=\frac{C^{\star}}{C}
\end{aligned}
$$

This definition of shadow costs is slightly different than the "gross" measure defined by Berndt and Fuss (1981) as $G+z_{k} K$, where the contribution of the quasi-fixed inputs are weighted by their shadow instead of rental values. The Berndt-Fuss expression derives, however, from a static optimization framework where the contribution of investment to costs is not explicitly defined. With dynamic optimization, shadow costs are "net" of adjustment costs because the costs of putting the marginal unit of capital into place are recognized. 5

Substituting (7) into (6) yields: 


$$
\varepsilon_{C t}^{\prime}=\frac{\varepsilon_{C t}}{1-\Sigma \varepsilon_{C j}-\sum \varepsilon_{\dot{C} j}}=\frac{\dot{y}}{y}-\sum \frac{w_{i} v_{i}}{C^{*}} \frac{\dot{v}_{i}}{v_{i}}-\sum \frac{z_{j} x_{j}}{C^{*}} \frac{\dot{x}_{j}}{x_{j}}-\sum \frac{z_{j} \dot{x}_{j}}{C} \frac{\ddot{x}_{j}}{\dot{x}_{j}} .
$$

In (8) $\varepsilon_{C t}^{\prime}$ is the expression for multifactor productivity with $\mathrm{CU}=1$, and is analogous to that derived by Berndt-Fuss; it adjusts observed productivity $\varepsilon_{\mathrm{Ct}}$ to account for subequilibrium. Essentially, (8) adjusts the shares so that the contribution of capital is weighted by its net shadow instead of rental value and it normalizes so that the sum of the resulting shares is equal to unity. There are three additional features of (8): (i) $\varepsilon_{\mathrm{Ct}}^{\prime}$ can be interpreted as the product of a conventional productivity measure and a $\mathrm{CU}$ term, (ii) $\varepsilon_{\mathrm{Ct}}^{\prime}$ can be shown to be equal to the production or primal side technical change measure adjusted for short run subequilibrium, $\varepsilon_{f t}^{\prime}$, and (iii) $\varepsilon_{C t}^{\prime}$ can directly be represented empirically using a parametric approach. I now pursue the first two points; the third is considered in the next section.

The value of the cost side measure, $C * / C$ or $\left(1-\Sigma \varepsilon_{C j}-\sum \varepsilon_{\dot{C}_{j}}\right)$, can lie on either side of unity depending on whether the net shadow valuation of the quasi-fixed inputs exceeds or falls short of the market rental price. 6 Thus the observed "residual" productivity change multiplied by $1 / C U=1 /\left(1-\Sigma \varepsilon_{C j}-\sum \varepsilon_{\dot{C}_{j}}\right)=C / C^{*}$ reflects the effect only of technical progress, which may be larger or smaller than the combined observed productivity effect.

Development of the production-side representation of technical progress requires demonstrating the equivalence of $\varepsilon_{C t}^{\prime}$ with a corresponding production-side measure $\varepsilon_{f t}^{\prime} \cdot$ First note that with 
fixed inputs the arguments of the production function must be divided into the variable and fixed inputs, here $v_{i}$ and $x_{j}$, so $Y=f\left(v_{i}, x_{j}, \dot{x}_{j}, t\right)$. Thus,

$$
\begin{aligned}
& \frac{d \ln Y}{d t}=\frac{\dot{y}}{Y}+\Sigma_{i} \frac{v_{i} f}{f} \frac{\dot{v}_{i}}{v_{i}}+\Sigma_{j} \frac{x_{j}^{f} j}{f} \frac{\dot{x}_{j}}{x_{j}}+\Sigma_{j} \frac{\dot{x}_{j}^{f \cdot} j}{f} \frac{\ddot{x}_{j}}{\dot{x}_{j}}+\varepsilon_{f t^{\prime}} \text { or, } \\
& \varepsilon_{f t}=\frac{\dot{Y}}{Y}-\Sigma_{i} \frac{v_{i} \mu f}{Y \mu} \frac{\dot{v}_{i}}{v_{i}}-\Sigma_{j} \frac{x_{j} \mu f}{Y \mu} \frac{\dot{x}_{j}}{x_{j}}-\Sigma_{j} \frac{\dot{x}_{j} \mu f \cdot j}{Y \mu} \frac{\ddot{x}_{j}}{\dot{x}_{j}} \text {. }
\end{aligned}
$$

If productivity growth $\varepsilon_{f t}$ is being measured from a point of subequilibrium, the quasi-fixed inputs must be revalued to represent the subequilibrium. This can be characterized by defining $\mu$ and $f_{j}$ from the firm's short run cost minimization problem:

(10) $\min C=\Sigma_{i} w_{i} v_{i}+\mu\left(Y-f\left(x_{j}, \dot{x}_{j}, v_{i}, t\right)\right)$.

Evaluation of (9) at the short run values derived from the first order conditions of (10) results in:

$$
\begin{aligned}
\varepsilon_{f t}^{i} & =\frac{\dot{y}}{Y}-\varepsilon_{C Y}^{-1}\left[\sum \frac{v_{i} w_{i}}{C} \frac{\dot{v}_{i}}{v_{i}}+\sum \frac{z_{j} x_{j}}{C} \frac{\dot{x}_{j}}{x_{j}}+\sum \frac{z_{j} \dot{x}_{j}}{C} \frac{\ddot{x}_{j}}{\dot{x}_{j}}\right] \\
& =\frac{\dot{Y}}{Y}-\frac{1}{1-\sum \varepsilon_{C j}-\sum \varepsilon_{\dot{C} j}}\left[\sum \frac{v_{i} w_{i}}{C} \frac{\dot{v}_{i}}{v_{i}}+\sum \frac{z_{j} x_{j}}{C} \frac{\dot{x}_{j}}{x_{j}}+\sum \frac{z_{j} \dot{x}_{j}}{C} \frac{\ddot{x}_{j}}{\dot{x}_{j}}\right]=\varepsilon_{C t}^{\prime} .
\end{aligned}
$$

This final expression is directly analogous to $\varepsilon_{C t}^{\prime}$ given by (8) above. Duality of $\varepsilon_{\mathrm{Ct}}$ and $\varepsilon_{\mathrm{ft}}$ in the CRTS case along with the equality between $\varepsilon_{f t}^{\prime}$ and $\varepsilon_{C t}^{\prime}$ together imply that $\varepsilon_{f t} / C U=\varepsilon_{f t}^{\prime}$ where $Y^{*} / \mathrm{Y}=1 / \mathrm{CU}$. Thus the output $\mathrm{CU}$ measure is exactly dual to the cost CU measure, provided long run CRTS and competition exist. 
In summary, in this integrated approach the observed productivity change $\varepsilon_{\mathrm{Ct}}$ is distorted from a true technical progress measure by fixed inputs and resulting deviations in $\mathrm{CU}$ from unity. It is possible, however, to inpute the true technical progress impact $\varepsilon_{\mathrm{Ct}}^{\prime}$ as the product of two parts, the observed productivity effect and the effect of short run rigidities or subequilibrium:

$$
\varepsilon_{f t}^{\prime}=\varepsilon_{f t} /\left(1-\sum \varepsilon_{C j}-\sum \varepsilon_{\dot{C}_{j}}\right) \text { or } \quad \varepsilon_{C t}^{\prime}=\varepsilon_{C t} /\left(1-\sum \varepsilon_{C j}-\sum \varepsilon_{\dot{C}_{j}}\right),
$$

where in the long run $\varepsilon_{\mathrm{Cj}}=\varepsilon_{\dot{\mathrm{C}}_{j}}=0$ so $\mathrm{CU}=1$ and $\varepsilon_{\mathrm{Ct}}^{\prime}=\varepsilon_{\mathrm{Ct}}=\varepsilon_{\mathrm{ft}}=\varepsilon_{\mathrm{ft}}^{\prime}$.

The cost-CU measure and its use as a link between short and long run productivity measures can be interpreted in various ways. ${ }^{7}$ one interpretation of this CU measure is as a form of a "multi-input Tobin's q" measure. Specifically, $\varepsilon_{C j}$ depends crucially on the deviation between $z_{j}$ and $p_{j}$, which is the basis for the calculation of marginal Tobin's $q$ as $\mathrm{z}_{\mathrm{k}} / \mathrm{p}_{\mathrm{k}}$, where $\mathrm{k}$ represents capital, and where only the stock effects or gross shadow value of capital is considered. Therefore in the case of one quasi-fixed input, adjustment by Tobin's $q$ as suggested by Berndt and Fuss is equivalent to multiplication by $\mathrm{CU}$. When there are multiple quasi-fixed inputs, while the weighting of different quasi-fixed inputs for Tobin's $\mathrm{q}$ is ambiguous, the $\mathrm{CU}$ adjustment is clearly appropriate.

Another interpretation of the cost $\mathrm{CU}$ measure is as a ratio of short and long run returns to scale, where short run returns to scale are defined over variable factors given fixed capital $=\varepsilon_{C Y}(s r)=\left(1-\sum \varepsilon_{C j}-\sum \varepsilon_{\dot{C}_{j}}\right)$. This view of $\mathrm{CU}$ is consistent with Ohta; in order to isolate technical 
change one must adjust by returns to scale, which in this case involves short run returns.

A final interpretation of the cost $\mathrm{CU}$ measure refers more to conventional methods of productivity measurement. Multiplication of the conventional measure by $\mathrm{C} / \mathrm{C}^{*}$ in effect revalues the shares of each of the inputs and, therefore, the weight on the change in that input to reflect the utilization of the given capital stock instead of the measured share. This multiplication incorporates the true economic valuation of the capital stock--its shadow marginal productivity in conjunction with the other inputs--instead of its simple market transaction value.

The CU adjustment therefore is important and is consistent with alternative interpretations. Observed productivity changes must also, however, be recognized to depend on the existence of nonstatic expectations, adjustment costs and NCRTS. I now consider these additions to the basic structure.

The conceptual importance of the extension to nonstatic expectations is that some current behavior of the firm may not correctly be imputed to responses in terms of current exogenous variables, but may result from anticipation of future economic conditions. Since the firm cannot respond instantaneously at a future point in time, it is rational for it to adjust partially before the exogenous change. Assuming that the observed productivity residual is a result only of rational optimizing firm behavior in terms of current variables may therefore omit important information that can further clarify the productivity residual. Introduction of nonstatic expectations introduces several 
complications. For example, with nonstatic expectations the economic agent is not striving toward "desired" levels of quasi-fixed input stocks $x^{*}(t)$ defined simply in terms of current variables, but instead can be envisaged as moving toward the sum of $x^{*}(t)$ and a discounted weighted average of the future desired stocks of $\mathbf{x}$ defined in terms of the anticipated paths of exogenous variables, $x^{* *}::^{8}$

$$
\dot{x}(t)=\lambda\left(x^{\star}-x+J\right)=\lambda\left(x^{\star *}-x\right) \quad 9^{9}
$$

In a model including nonstatic expectations the effect of $\mathrm{J} \neq 0$ on the $\mathrm{CU}$ measure can be captured by imputing the current $\dot{x}$ that would allow a geometric progression to $x^{*}$ instead of to $x^{* *}$, and then determining the levels of variable inputs necessary to produce actual output and these imputed investment levels, instead of those including all anticipatory behavior. Two alternative CU measures can then be calculated, (i) a current value measure $\mathrm{CU}_{\mathrm{CV}^{\prime}}$ and (ii) an imputed present value measure $\mathrm{CU}_{\mathrm{pv}}{ }^{\cdot}$ The difference between these can be attributed to anticipatory behavior.

$\mathrm{CU}_{\mathrm{CV}}$ is based on measurement within the full nonstatic expectations model and includes both subequilibrium and nonstatic expectations effects. Calcualtion of $\mathrm{CU}_{\mathrm{pv}}$ requires purging from $\varepsilon_{\mathrm{Cj}}$ and $\varepsilon_{\dot{C} j}$ for all $j$ the effects of investment due purely to anticipatory behavior, rather than that attributed to current exogenous variables. This results in the adjusted CU measure $\left(1-\sum \varepsilon_{\mathrm{Cj}}-\sum \varepsilon_{\dot{C}_{j}}\right)^{\prime}=\mathrm{CU}_{\mathrm{pv}} \cdot$ Adjustment of a conventional productivity growth measure by dividing by (1- $\left.-\Sigma \varepsilon_{C j}-\Sigma \varepsilon_{C_{j}}\right)$ has been interpreted as revaluing input shares to reflect the full utilization of the quasi-fixed inputs including all 
observed variable input use. By contrast, adjustment by (1 $\left.-\Sigma \varepsilon_{C j}-\Sigma \varepsilon_{\dot{C}_{j}}\right)^{\prime}$ incorporates the idea that variable inputs used for anticipatory investment should not be evaluated as if they were contributing to current productivity. Note that this procedure for purging the effects of anticipatory behavior, although it attributes all current variable input demand to decisions based on current variables, does not categorize all variable input demands as those corresponding to current output production. To sharpen measures of technical change it is however necessary to purge costs of adjustment corresponding to all investment, not just anticipatory investment, from the productivity calculation. To accomplish this, $\left(1-\sum \varepsilon_{C j}-\sum \varepsilon_{\dot{C}_{j}}\right) "$ or $C_{\text {net }}$ can be derived as a subequilibrium indicator net of all investment costs that distort measurement of cost diminution. By construction this measure purges the shares of variable inputs of all input use that is not directly productive in terms of augmenting current output levels.

The final adjustment of productivity measures requires incorporation of NCRTS. The importance of this extension in the preceding analysis based on CRTS is first evident in equation (3); the substitution of (1- $\left.-\sum \varepsilon_{C j}-\sum \varepsilon_{C_{j}}\right)$ for $\varepsilon_{C Y}$ is valid only with CRTS. To see this, note that:

$$
\frac{d \ln C}{d \ln Y}=\frac{Y}{C}\left[\frac{\partial C}{\partial Y}+\Sigma_{j} \frac{\partial C}{\partial x_{j}} \frac{d x_{j}}{d Y}+\Sigma_{j} \frac{\partial C}{\partial \dot{x}_{j}} \frac{d \dot{x}_{j}}{d Y}\right]
$$

With nonconstant returns to scale, $d \ln C / d \ln Y=\eta$ where $1 / \eta$ captures long run returns to scale, and $d \ln x_{j} / d \ln Y=d \ln \dot{x}_{j} / d \ln Y=\eta_{j} \neq \eta \neq 1$. Thus (14) reduces to: 


$$
n=\varepsilon_{C Y}+\Sigma_{\eta_{j}}\left(\varepsilon_{C j}+\varepsilon_{\dot{C}_{j}}\right) \quad \text { or } \quad \varepsilon_{C Y}=n\left[1-\Sigma_{j}\left(\frac{\eta_{j}}{n}\right)\left(\varepsilon_{C j}+\varepsilon_{\dot{C}_{j}}\right)\right]
$$

If the cost function is homothetic, $\varepsilon_{C Y}=n\left(1-\sum \varepsilon_{C j}-\sum \varepsilon_{\dot{C}_{j}}\right)$. In this case overall returns to scale are decomposed into two parts, a subequilibrium component, $\left(1-\Sigma \varepsilon_{C j}-\Sigma \varepsilon_{C_{j}}\right)$ and a returns to scale component, $n$. Adjusting the observed productivity measure represented by (5) for NCRTS then yields

$$
\varepsilon_{C t}=n\left(1-\Sigma \varepsilon_{C j}-\sum \varepsilon_{\dot{C}_{j}}\right) \frac{\dot{y}}{Y}-\sum \frac{w_{i} v_{i}}{C} \frac{\dot{v}_{i}}{v_{i}}-\sum \frac{z_{j} x_{j}}{C} \frac{\dot{x}_{j}}{x_{j}}-\frac{z \cdot \dot{x}_{j}}{C} \frac{\ddot{x}_{j}}{\dot{x}_{j}} .
$$

The adaptation of $\varepsilon_{\mathrm{Ct}}$ for subequilibrium is analogous to the CRTS case since CU represents short run relative to long run returns to scale, or $n\left(1-\Sigma \varepsilon_{C j}-\Sigma \varepsilon_{\dot{C}_{j}}\right) / n=1-\Sigma \varepsilon_{C j}-\sum \varepsilon_{\dot{C}_{j}}$ (see Morrison (1983c)). Thus:

$$
\begin{aligned}
\varepsilon_{C t}^{\prime}=\frac{\varepsilon_{C t}}{1-\sum \varepsilon_{C j}-\sum \varepsilon_{\dot{C} j}}=n \frac{\dot{y}}{y}-\frac{1}{1-\sum \varepsilon_{C j}-\sum \varepsilon_{C_{j}}}\left[\sum \frac{w_{i} v_{i}}{C} \frac{\dot{v}_{i}}{v_{i}}\right. & +\sum \frac{z_{j} x_{j}}{C} \frac{\dot{x}_{j}}{x_{j}} \\
& \left.+\sum \frac{z_{j} \dot{x}_{j}}{C} \frac{\ddot{x}_{j}}{\dot{x}_{j}}\right]
\end{aligned}
$$

or,

$$
\frac{\varepsilon_{C t}^{\prime}}{n}=\frac{\dot{y}}{y}-\frac{1}{n\left(1-\Sigma \varepsilon_{C j}-\Sigma \varepsilon_{C j}\right)}\left[\sum \frac{w_{i} v_{i}}{c} \frac{\dot{v}_{i}}{v_{i}}+\sum \frac{z_{j} x_{j}}{c} \frac{\dot{x}_{j}}{x_{j}}+\sum \frac{z_{j} \dot{x}_{j}}{C} \frac{\ddot{x}_{j}}{\dot{x}_{j}}\right] .
$$

Note that from (15), since $\varepsilon_{C Y}$ equals $n\left(1-\Sigma \varepsilon_{C j}-\Sigma \varepsilon_{\dot{C}_{j}}\right)$, (18) in turn equals $\varepsilon_{C t}^{\prime} \cdot$ It follows that:

$$
\frac{\varepsilon_{C t}^{\prime}}{n}=\frac{\varepsilon_{C t}}{\left(1-\Sigma \varepsilon_{C j}-\sum \varepsilon_{\dot{C}_{j}}\right) n}=\frac{\varepsilon_{f t}}{\left(1-\sum \varepsilon_{C j}-\sum \varepsilon_{\dot{C}_{j}}\right)}=\varepsilon_{f t}^{\prime} .
$$


In the nonhomothetic case, as discussed in Morrison (1983c) the CU measure is not simply ( $\left.1-\sum \varepsilon_{\mathrm{Cj}}-\sum \varepsilon_{\dot{C}_{j}}\right)=$ SHCOST/TCOST but must instead be adjusted by differential scale effects for different inputs, as follows:

$$
\begin{aligned}
\frac{\varepsilon_{C t}^{\prime}}{n}=\frac{\varepsilon_{C t}}{\eta\left[1-\frac{\sum \eta_{j}\left(\varepsilon_{C j}+\varepsilon_{C j}\right)}{\eta}\right]}=\frac{\dot{y}}{Y}-\frac{1}{\eta\left[1-\frac{\sum \eta_{j}\left(\varepsilon_{C j}+\varepsilon_{C_{j}}\right)}{\eta}\left[\sum \frac{{ }_{i} v_{i}}{C} \frac{\dot{v}_{i}}{v_{i}}\right.\right.} \\
\left.+\sum \frac{z_{j} x_{j}}{C} \frac{\dot{x}_{j}}{x_{j}}+\Sigma \frac{z_{j} \dot{x}_{j}}{C} \frac{\ddot{x}_{j}}{\dot{x}_{j}}\right] \\
=\frac{\varepsilon_{C t}}{C U \cdot \eta}=\frac{\varepsilon_{f t}}{C U}=\varepsilon_{f t}^{\prime}
\end{aligned}
$$

With nonconstant returns to scale, the shares are thus adjusted to reflect the fact that some increases in "efficiency" or decreases in cost are simply due to scale effects. The NCRTS adjustment alters the cost shares to represent output shares--purging the share of the scale effect that decreases costs with no true increase in input efficiency. 


\section{EMPIRICAL ILLUSTRATION}

I now turn to an empirical implementation of the decomposition of the path of productivity growth over time. Specifically, I attempt to assess empirically the role of subequilibrium, adjustment costs, nonstatic expectations, and NCRTS in determining the 1949-1979 path of productivity growth in U.S. manufacturing. 10 I proceed first to summarize the measures used to decompose observed productivity growth and to isolate technical progress.

The measure of productivity used as a reference point is $\varepsilon_{\mathrm{Ct}}$. This is the conventional measure of cost-side total factor productivity calculated from the data on actual cost shares and changes in cost and input prices as the (negative) difference between $\dot{C} / C$ and the shareweighted sum of input prices $\dot{w}_{i} / w_{i}$ and $\dot{p}_{j} / p_{j}$. It is also a justifiable measure of productivity growth given long run equilibrium, instantaneous adjustment, static expectations, and CRTS. The decomposition of $\varepsilon_{C t}$ is carried out by generalizing each of these restrictions. For expositional purposes, to represent $\varepsilon_{C t}$ I rely on the conventional index of the state of technology or level of productivity denoted $A_{t}$, where $\varepsilon_{C t}=\dot{A} / A$.

Some of the observed productivity residual $\varepsilon_{C t}$ may arise simply from short run "rigidities" rather than from technical progress. A more appropriate measure to isolate the impact of subequilibrium has been shown to be $\varepsilon_{\mathrm{Ct}}^{\prime}=\varepsilon_{\mathrm{Ct}} / \mathrm{CU}^{+}=\varepsilon_{\mathrm{Ct}} / \mathrm{CU}_{\mathrm{pv}}$; the corresponding productivity index is denoted $A_{t}^{\prime}$. This is the first adjustment to be analyzed below.

In addition, observed changes in productivity may arise from 
nonstatic expectations. To adjust for nonstatic expectations it is sufficient simply to measure $\varepsilon_{\mathrm{Ct}}^{\prime \prime}=\varepsilon_{\mathrm{Ct}} / \mathrm{CU}_{\mathrm{CV}^{\prime}}$ and the resulting state of technology $A_{t}^{\prime \prime} \cdot C U_{C V}$ is a temporary equilibrium indicator based on current instead of intertemporal optimization. This is the second adjustment to be discussed.

Since some observed productivity may also result from fluctuations in variable input use for investment, the corresponding adjustment costs should be purged in order to derive a sharper measure of technical progress. This is accomplished by calculating $\varepsilon_{\mathrm{Ct}}^{\prime \prime \prime}=\varepsilon_{\mathrm{Ct}} / \mathrm{CU}_{\text {net }}$, and cumulating these growth rates to derive the index of productivity levels, $A_{t}^{\prime \prime}$ '. This is the third adjustment to be assessed.

This third measure, however, reflects true technical progress only if the technology exhibits CRTS. Otherwise, the apparent productivity changes resulting from scale effects are incorrectly attributed to technical progress. To adjust for this it is necessary to multiply by returns to scale, or in the notation above, to calculate $\varepsilon_{\mathrm{Ct}}^{\prime \prime \prime}=\varepsilon_{\mathrm{Ct}}^{\prime \prime \prime} / \eta$ and the associated $A_{t}^{\prime \prime \prime \prime}$. This final expression for $\varepsilon_{C t}$ is the technical progress residual after adjusting for the full set of structural and behavioral factors considered above. It may therefore be used to determine whether, during periods of volatility, changes in the economic situation usually attributed simply to "productivity" are instead attributable to other direct and indirect impacts.

The model I use to construct and estimate these measures is from Morrison (1983b). Briefly, this model assumes capital is quasi-fixed and that costs of adjustment occur for net capital formation. An 
alternative specification compared here also includes nonproduction workers (N) as a quasi-fixed factor; labor is "hoarded" in the sense that there are costs of adjustment on net investment in the "stock" of nonproduction workers. The firm is assumed to produce output using capital and three variable inputs, labor (L) (or production workers (P) for the alternative specification), energy (E), and nonenergy intermediate materials (M). The firm's optimization process involves maximizing at each time $t$ the present value of the future stream of profits given expected paths of the exogenous variables--output demand and input prices--and adjustment cost relations. This is accomplished sequentially (as in Morrison (1983a)) by minimizing variable costs for any output and quasi-fixed input levels, setting output price according to a short run condition of equality between marginal revenues and costs to determine the output level, and finally by choosing an optimal investment plan, conditional on the quasi-fixed input stocks at time $t$. The firm's decisions are represented, given an explicit form for the variable cost function $G$, by a system of demand equations for its variable inputs, an output price equation, and investment equations analogous to (13) for the quasi-fixed inputs incorporating nonstatic expectations. Parameter estimation by maximum likelihood procedures results in a full specification of short and long run elasticities. Specifically, the model permits calculation of $\mathrm{CU}, \varepsilon_{\mathrm{Ct}}{ } \varepsilon_{\mathrm{Cj}}{ }^{\prime} \varepsilon_{\dot{C}_{j}}$, and $\varepsilon_{\mathrm{CY}}{ }^{11}$

In the first column of Table $1 \mathrm{~A}$, I report the conventionally measured index of the state of technology $A_{t} \cdot$ Productivity indices calculated from the adjusted productivity growth estimates are presented 
in the next four columns for the one quasi-fixed input model as $A_{t}^{\prime}$ ' $A_{t}^{\prime \prime}, A_{t}^{\prime \prime \prime}$, and $A_{t}^{\prime \prime \prime}$. Analogous productivity measures for the alternative two quasi-fixed input model, recognizing the short run fixity of both $K$ and $N$, are presented in Table $1 B$.

Although these conventional year-by-year measures provide some indication of the post-1973 productivity slowdown, the impacts of the adjustments are not immediately evident. I therefore summarize the information contained in Tables $1 \mathrm{~A}$ and $\mathrm{IB}$ using average annual productivity growth rates over selected time periods; these are presented in Table 2. This temporal breakdown is based on the common divisions into time periods of pre-1965, 1965 to 1973, and post-1973.

The first point to note from Table 2 is that the measures calculated at the two levels of labor aggregation differ slightly. Over the entire time period 1949-1979, the traditionally measured average annual multifactor productivity increase measured using four inputs, $\mathrm{K}, \mathrm{L}, \mathrm{E}$, and $M$, was .838 per year, whereas once $L$ is disaggregated into $N$ and $P$ productivity growth over this period appears one tenth larger--.90\%/year. The post-1965 slowdown also appears more noticeable when both nonproduction and production workers are considered separately than when they are aggregated. This difference is largest precisely when the greatest compositional changes occurred within $L$; such changes are obscured by aggregation.

Overall, from either model, a substantial amount of productivity decline is evident since 1949-1965. A great deal of the productivity growth change, however, appears to have taken place before 1973, as was also noted by Norsworthy et al. (1979). In particular, although from 
Table IA

Total Factor Productivity Indices, One Quasi-Fixed Input Model U.S. Manufacturing, $1972=1.0$

\begin{tabular}{|c|c|c|c|c|c|}
\hline Year & $\mathrm{A}_{\mathrm{t}}$ & $A_{t}^{\prime}$ & $A_{t}^{\prime \prime}$ & $A_{t}^{\prime \prime \prime}$ & $A_{t}^{\prime \prime \prime \prime}$ \\
\hline 1949 & .8149 & .8197 & .8176 & .8116 & .8255 \\
\hline 1950 & .8356 & .8400 & .8381 & .8327 & .8457 \\
\hline 1951 & .8518 & .8560 & .8541 & .8490 & .8613 \\
\hline 1952 & .8552 & .8593 & .8574 & .8524 & .8645 \\
\hline 1953 & .8660 & .8699 & .8680 & .8633 & .8749 \\
\hline 1954 & .8613 & .8653 & .8634 & .8586 & .8705 \\
\hline 1955 & .8871 & .8904 & .8888 & .8849 & .8951 \\
\hline 1956 & .8795 & .8829 & .8813 & .8771 & .8880 \\
\hline 1957 & .8742 & .8778 & .8762 & .8720 & .8831 \\
\hline 1958 & .8896 & .8931 & .8915 & .8872 & .8972 \\
\hline 1959 & .8953 & .8987 & .8971 & .8928 & .9024 \\
\hline 1960 & .9141 & .9170 & .9153 & .9112 & .9191 \\
\hline 1961 & .9172 & .9200 & .9183 & .9143 & .9218 \\
\hline 1962 & .9418 & .9435 & .9419 & .9385 & .9435 \\
\hline 1963 & .9396 & .9415 & .9399 & .9364 & .9416 \\
\hline 1964 & .9627 & .9632 & .9624 & .9603 & .9632 \\
\hline 1965 & 9699 & .9700 & .9696 & .9680 & .9704 \\
\hline 1966 & .9606 & .9613 & .9604 & .9582 & .9613 \\
\hline 1967 & .9596 & .9603 & .9594 & .9571 & .9603 \\
\hline 1968 & .9702 & .9705 & .9699 & .9682 & .9706 \\
\hline 1969 & .9745 & .9748 & .9742 & .9727 & .9747 \\
\hline 1970 & .9636 & .9637 & .9631 & .9615 & .9644 \\
\hline 1971 & .9782 & .9785 & .9781 & .9770 & .9788 \\
\hline 1972 & 1.0000 & 1.0000 & 1.0000 & 1.0000 & 1.0000 \\
\hline 1973 & 1.0262 & 1.0254 & 1.0265 & 1.0284 & 1.0266 \\
\hline 1974 & 1.0155 & 1.0148 & 1.0158 & 1.0172 & 1.0165 \\
\hline 1975 & 1.0078 & 1.0070 & 1.0079 & 1.0088 & 1.0088 \\
\hline 1976 & 1.0258 & 1.0251 & 1.0267 & 1.0291 & 1.0278 \\
\hline 1977 & 1.0408 & 1.0399 & 1.0421 & 1.0458 & 1.0436 \\
\hline 1978 & 1.0474 & 1.0463 & 1.0492 & 1.0536 & 1.0513 \\
\hline 1979 & 1.0546 & 1.0536 & 1.0572 & 1.0626 & 1.0604 \\
\hline
\end{tabular}


Table IB

Total Factor Productivity Indices, Two Quasi-Fixed Input Model U.S. Manufacturing, $1972=1.0$

\begin{tabular}{|c|c|c|c|c|c|}
\hline Year & ${ }_{t}$ & $A_{t}^{\prime}$ & $A_{t}^{\prime \prime}$ & $A_{t}{ }_{t}^{\prime \prime}$ & $A_{t}^{\prime}{ }^{\prime \prime \prime \prime}$ \\
\hline 1949 & .7872 & .7872 & .7835 & .7794 & .7921 \\
\hline 1950 & .8018 & .8009 & .7977 & .7939 & .8062 \\
\hline 1951 & .8214 & .8195 & .8167 & .8135 & .8249 \\
\hline 1952 & .8322 & .8299 & .8270 & .8240 & .8346 \\
\hline 1953 & .8438 & .8410 & .8380 & .8354 & .8450 \\
\hline 1954 & .8488 & .8458 & .8431 & .8406 & .8501 \\
\hline 1955 & .8695 & .8658 & .8629 & .8610 & .8686 \\
\hline 1956 & .8658 & .8622 & .8593 & .8574 & .8654 \\
\hline 1957 & .8659 & .8614 & .8585 & .8565 & .8646 \\
\hline 1958 & .8905 & .8879 & .8863 & .8838 & .8909 \\
\hline 1959 & .8914 & .8888 & .8872 & .8847 & .8917 \\
\hline 1960 & .9148 & .9126 & .9115 & .9093 & .9143 \\
\hline 1961 & .9192 & .9171 & .9159 & .9138 & .9183 \\
\hline 1962 & .9439 & .9419 & .9406 & .9392 & .9411 \\
\hline 1963 & .9415 & .9395 & .9383 & .9368 & .9391 \\
\hline 1964 & .9673 & .9651 & .9656 & .9645 & .9666 \\
\hline 1965 & .9674 & .9652 & .9657 & .9646 & .9667 \\
\hline 1966 & .9591 & .9570 & .9574 & .9562 & .9586 \\
\hline 1967 & .9629 & .9608 & .9613 & .9603 & .9626 \\
\hline 1968 & .9758 & .9743 & .9738 & .9732 & .9740 \\
\hline 1969 & .9808 & .9796 & .9786 & .9781 & .9782 \\
\hline 1970 & .9768 & .9750 & .9739 & .9734 & .9737 \\
\hline 1971 & .9875 & . 9869 & .9870 & .9868 & .9876 \\
\hline 1972 & 1.0000 & 1.0000 & 1.0000 & 1.0000 & 1.0000 \\
\hline 1973 & 1.0190 & 1.0194 & 1.0178 & 1.0185 & 1.0164 \\
\hline 1974 & 1.0018 & 1.0012 & .9999 & .9996 & .9990 \\
\hline 1975 & 1.0022 & 1.0018 & 1.0004 & 1.0001 & .9996 \\
\hline 1976 & 1.0175 & 1.0187 & 1.0178 & 1.0181 & 1.0180 \\
\hline 1977 & 1.0289 & 1.0308 & 1.0304 & 1.0312 & 1.0316 \\
\hline 1978 & 1.0329 & 1.0351 & 1.0348 & 1.0356 & 1.0365 \\
\hline 1979 & 1.0391 & 1.0420 & 1.0416 & 1.0426 & 1.0441 \\
\hline
\end{tabular}


Table 2

Average Total Factor Productivity Growth Rates Observed and Adjusted, U.S. Manufacturing, Selected Periods

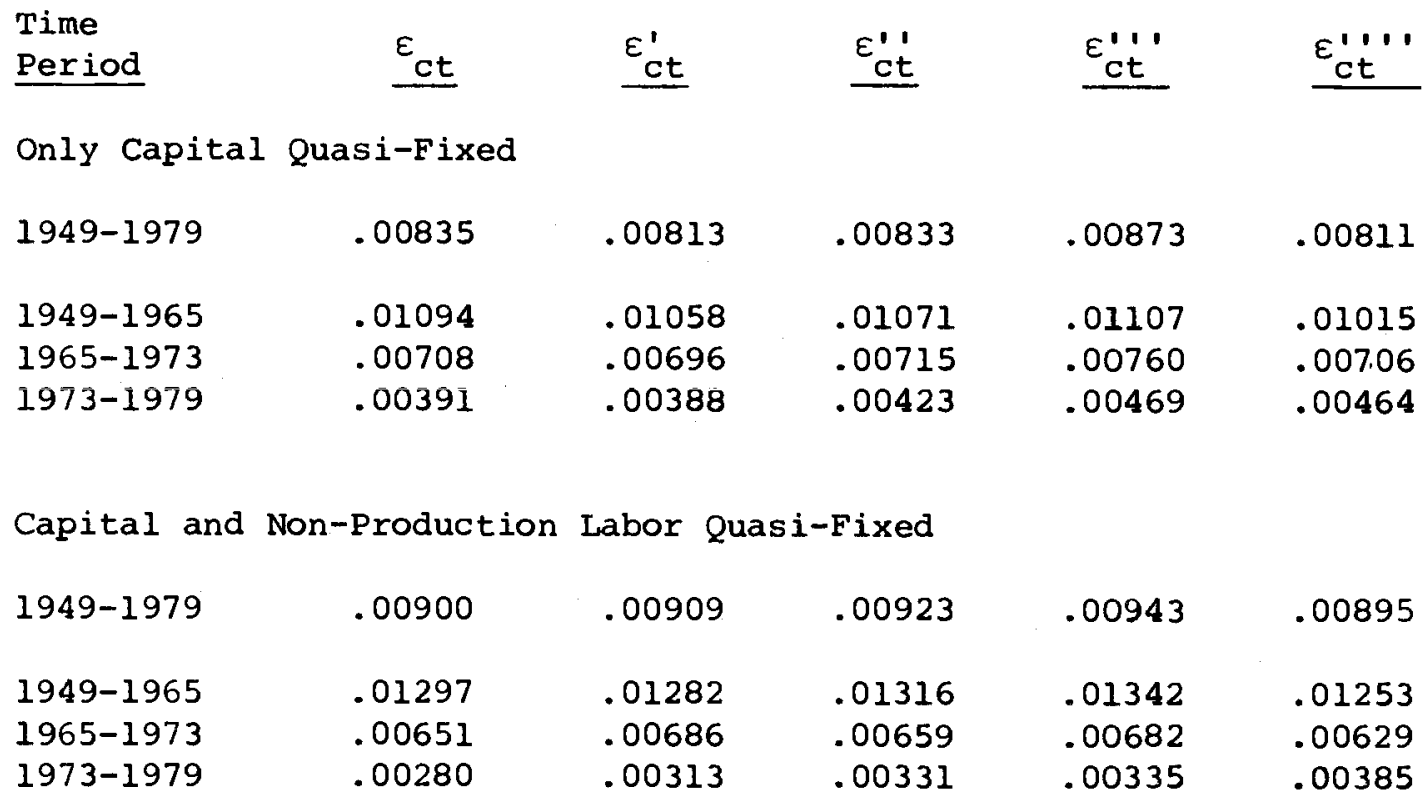

Key: Alternative residual $\varepsilon_{c t}$ Measures Based on Assumptions of: $\varepsilon_{c t}=$ residual measure: instantaneous adjustment, static expectations, no costs of adjustment, and CRTS

$\varepsilon_{c t}^{\prime}=\varepsilon_{c t} / \mathrm{CU}_{\mathrm{pv}}$ : static expectations, no costs of adjustment and CRTS

$\varepsilon_{c t}^{\prime \prime}=\varepsilon_{c t} / \mathrm{CU}{ }_{C V}:$ no costs of adjustment and CRTS

$\varepsilon_{c t}^{\prime \prime \prime}=\varepsilon_{c t} / \mathrm{CU}$ net $:$ CRTS

$\varepsilon_{c t}^{\prime \prime \prime}=\varepsilon_{c t}^{\prime \prime \prime} / n:$ Final 
1949-1965 the average annual growth rate (AAGR) was 1.18 to 1.38 , between 1965 and 1973 it dropped to .658 to $.70 \%$. From Tables $1 \mathrm{~A}$ and 1B, this appears to be a result of two significant declines in productivity, one in 1966-1967 and another in 1970. Thus the AAGR over the subperiod from 1965-1970 was negative--approximately -.18 to -.28 . This period could be a strong contender for the title of "productivity growth slowdown."

Compared to the 1965-1973 period, post 1973 the AAGR dropped further, to approximately .41 to $.448--o n l y$ about 358 of the productivity growth rate observed in 1949-1965. This reflects, of course, the celebrated recent "productivity growth slowdown." This productivity growth slowdown is, however, driven by the productivity decreases of only a couple of years. If, for example, a researcher in 1977 were considering the productivity decline from 1973 it would appear much more imposing; the 1973-1976 period exhibited a slight productivity decrease of approximately -.018 per year. From 1976 on the AAGR in productivity began to attenuate this evidence of stagnation; total factor productivity between 1976 and 1979 again began to grow at about .78 to .98 per year, close to that occurring between 1949 and 1965.

Hence there appear to be two periods when large downward shifts in productivity growth occurred, namely, the late 1960's and the few years subsequent to 1973. Together these result in a rather substantial overall effect on 1965-1979 productivity growth as compared to the previous fifteen years, but they do not present conclusive evidence of a unique sudden slacking off of productivity growth post-1973 in U.S. manufacturing. These trends in observed productivity growth are attenuted somewhat 
by the impact of the adjustments outlined above. The first adjustment to the productivity measure is that for subequilibrium. The empirical significance of this adjusted productivity measure, reported as $A_{t}^{\prime}$ in Table 1 and in terms of AAGR as $\varepsilon_{c t}^{\prime}$ in Table 2, differs across models. With the adjustment, in the one quasi-fixed input model the residual decreases in all sub-periods, but by the largest proportion in the earlier years; part of the relatively large growth in productivity observed in earlier periods appears to have been due to the effects of subequilibrium. For the two quasi-fixed input model productivity growth appears larger for both later time periods for $A_{t}^{\prime}$ than for ${ }^{A} t^{\prime}$ particularly for the 1973-1979 time period. This constitutes evidence on the role of labor hoarding as well as fixity of capital in accounting for subequilibrium effects.

The above results reveal a modest decrease in the dispersion of productivity measures over time with the subequilibrium adjustment. In addition, the late 1960s still appears as a period of poor productivity performance and the two years after 1973 seem ever more catastrophic as a result of large unexpected shocks, particularly for the two quasifixed input model.

The second adaptation of the productivity growth measure to $\varepsilon_{c t}^{\prime \prime}$ is more dramatic. The adjustment for nonstatic expectations in the one quasi-fixed input model results in a larger productivity residual for all time periods--even larger than the unadjusted $\varepsilon_{\mathrm{ct}}$ measure for the two later time periods. This suggests a tendency for anticipatory investment to have a depressing effect on traditional productivity growth measures. This same tendency is evident for the two quasi-fixed input model. The distincition between subequilibrium adjustment to $\varepsilon_{c t}^{\prime}$ and the further nonstatic expectations adjustment to $\varepsilon_{c t}^{\prime \prime}$ is that the former 
reveals the impact of unexpected subequilibrium which is not properly accommodated--subequilibrium occurs in terms of present as well as current valued variables--whereas the latter documents the effect of anticipated changes. This implies that although when the firm is optimistic productivity measures adjusted for nonstatic expectations likely improve, at times when the firm's expectations are incorrect $\varepsilon_{\text {ct }}^{\prime \prime}$ may fall. To illustrate the implications of this, reconsider the indices in Tables $1 \mathrm{~A}$ and 1B. Although E' $E_{\text {Ct }}^{\prime \prime}$ between 1973 and 1976 appears uniformly better than $\varepsilon_{c t}^{\prime}$, events in 1974 still had very depressing effects as firms attempted to adjust to a subequilibrium resulting from unanticipated shocks in 1973.

The revision to account for total adjustment costs of $\varepsilon_{c t}^{\prime \prime}$ to E''' simply augments, as expected, the trends found for the expectations adjustment. Specifically, when net investment corresponding to current exogenous variables is also purged, so that input use only for produced output and not investment is attributed to the productivity measure, productivity appears more robust over the entire period. In total, over the 1949-1979 period the combined effect of these revisions to $\varepsilon_{c t}$ in the two quasi-fixed input model indicates that productivity growth was approximately .948 per annum in contrast to the $.90 \%$ corresponding to contemporaneous observations. This tendency is even more pronounced for the final sub-period. The unadjusted measure indicates .28\% AAGR over the 1973-1979 period, whereas with the three adaptations the AAGR becomes $.335 \%$, about a $20 \%$ increase. Results for the one quasi-fixed input model are similar.

Additional information about the first three adjustments may be 
obtained by considering the alternative CU indicators directly. These indices are presented in Table 3 as $\mathrm{CU}_{\mathrm{pv}^{\prime}} \mathrm{CU}_{\mathrm{Cv}^{\prime}}$ and $\mathrm{CU}_{\text {net }}$ respectively for the two models. The most obvious difference among the indices is that the $\mathrm{CU}_{\mathrm{CV}}$ and $\mathrm{CU}_{\text {net }}$ indicators reach values above unity less often than the $\mathrm{CU}_{\mathrm{pv}}$ measure. As discussed further in Morrison (1984), this is a priori appealing; CU indicators including nonstatic expectations tend to fall short of unity any time the firm is investing additionally in anticipation of, for example, output increases not justified on the basis of current economic conditions. Comparison of the overall patterns of the $\mathrm{CU}$ indices yields several other interesting implications. Consider the "preferred" two quasi-fixed input model $\mathrm{CU}$ measures. In the first few years and in the early 1960s $\mathrm{CU}_{\mathrm{pv}}>1>\mathrm{CU}_{\text {net' }}$ indicating optimistic investment and a shortage of capacity in terms of present value investment, even though current observation indicates excess capacity. In 1952 to 1953, 1955 to 1956 and 1963 there is a shortage of capacity in both present and current value terms, inducing additional investment. In the late 1950s and from 1967 on both $\mathrm{CU}_{\mathrm{pv}}$ and $\mathrm{CU}_{\mathrm{Cv}}$ tend to fall short of unity, although $\mathrm{CU}_{\mathrm{pv}}$ is typically closer to one. This indicates that although firms were optimistic about the future their optimism justified only cautious investment plans, since capacity was underutilized even in terms of present value. Finally, in 1969 and 1973 pessimism appears to have been prevalent; $\mathrm{CU}_{\mathrm{pv}}<1<\mathrm{CU}_{\mathrm{CV}^{\prime}}$ indicating a currently observed shortage of capacity which actually corresponds to a slight excess of available capacity given expectations of future conditions. The numbers for the one quasi-fixed input model are slightly different; they tend in 
Table 3

Capacity Utilization Indices

U.S. Manufacturing

\begin{tabular}{|c|c|c|c|c|c|c|}
\hline Year & ${ }^{\mathrm{CU}} \mathrm{pv}_{\mathrm{p}}$ & $\mathrm{CU}_{\mathrm{CV}}$ & $\mathrm{Cu}_{\text {net }}$ & $\mathrm{CU}_{\mathrm{pv}}$ & $\mathrm{Cu}_{\mathrm{CV}}$ & $\mathrm{CU}_{\text {net }}$ \\
\hline & One & Quasi-Fixed & Input & Two $\&$ & Quasi-Fixed & Inputs \\
\hline 1949 & 1.0225 & 1.0198 & . 9989 & 1.0595 & .9968 & .9741 \\
\hline 1950 & 1.0232 & 1.0135 & .9807 & 1.0594 & 1.0227 & .9900 \\
\hline 1951 & 1.0243 & 1.0188 & .9898 & 1.0504 & 1.0216 & .9910 \\
\hline 1952 & 1.0247 & 1.0168 & .9888 & 1.0402 & 1.0475 & 1.0230 \\
\hline 1953 & 1.0272 & 1.0192 & .9917 & 1.0439 & 1.0503 & 1.0092 \\
\hline 1954 & 1.0241 & 1.0195 & .9934 & 1.0195 & .9644 & .9488 \\
\hline 1955 & 1.0310 & 1.0155 & .9762 & 1.0340 & 1.0406 & 1.0028 \\
\hline 1956 & 1.0302 & 1.0184 & .9786 & 1.0155 & 1.0396 & 1.0046 \\
\hline 1957 & 1.0206 & 1.0208 & 1.0149 & .9924 & .9793 & .9662 \\
\hline 1958 & 1.0083 & 1.0084 & 1.0097 & .9579 & .9098 & .9261 \\
\hline 1959 & 1.0211 & 1.0198 & 1.0024 & .9842 & 1.0128 & .9914 \\
\hline 1960 & 1.0304 & 1.0307 & 1.0183 & .9801 & .9600 & .9459 \\
\hline 1961 & 1.0321 & 1.0313 & 1.0081 & .9755 & .9871 & .9739 \\
\hline 1962 & 1.0466 & 1.0439 & 1.0110 & .9942 & .9953 & .9673 \\
\hline 1963 & 1.0539 & 1.0409 & .9927 & 1.0049 & 1.0346 & 1.0039 \\
\hline 1964 & 1.0614 & 1.0231 & .9606 & 1.0097 & .9438 & .9291 \\
\hline 1965 & 1.0069 & .9983 & .9351 & 1.0304 & .9396 & .9519 \\
\hline 1966 & 1.0604 & 1.0024 & .9371 & 1.0013 & .9930 & .9879 \\
\hline 1967 & 1.0444 & 1.0173 & .9620 & .9710 & .9582 & .9314 \\
\hline 1968 & 1.0307 & 1.0000 & .9448 & .9566 & 1.0300 & .9986 \\
\hline 1969 & 1.0232 & 1.0096 & .9651 & .9405 & 1.0584 & 1.0109 \\
\hline 1970 & .9892 & .9863 & .9708 & .8815 & .8721 & .8719 \\
\hline 1971 & .9884 & .9756 & .9374 & .9007 & .8169 & .8070 \\
\hline 1972 & 1.0162 & .9966 & .9498 & .9543 & .9622 & .9428 \\
\hline 1973 & 1.0324 & .9906 & .9237 & .9759 & 1.0651 & 1.0233 \\
\hline 1974 & 1.0192 & 1.0111 & .9641 & .9456 & .9587 & .9075 \\
\hline 1975 & .9806 & .9644 & .9112 & .8726 & .8234 & .8241 \\
\hline 1976 & .9956 & .9589 & .8904 & .9070 & .8799 & .8509 \\
\hline 1977 & 1.0148 & .9716 & .9001 & .9377 & .9024 & .8733 \\
\hline 1978 & 1.0136 & .9289 & .8439 & .9361 & .9210 & .9041 \\
\hline 1979 & .9996 & .9041 & .8079 & .9050 & .9122 & .8905 \\
\hline
\end{tabular}


general to be higher, and do not capture such phenomena as the pessimism of 1969 and 1973.

The last productivity adjustment to consider is that for NCRTS. The AAGR for $\varepsilon_{\text {ct }}^{\prime \prime \prime}$ is the final column of Table 2 indicate that although overall productivity growth estimates 1949-1979 are substantial with effects of scale economies purged, this is not true for 1973-1979. In fact for the two quasi-fixed input case the reverse is true for 1973-1979. The difference in growth rates for both models between the earlier and later years is diminished further with this adaptation.

Recall that this scale adjustment recognizes the difference between decreasing costs due to (i) pure technical progress and (ii) scale economies. Over the 1949-1973 time period on average both of these effects were evident. As a result, removing the scale effect imputes a lower growth rate to technical progress. However, when output grows at a lower or negative rate, the reverse can occur, i.e., lower productivity growth is incorrectly attributed to reductions in the growth rate of technical progress. During the sluggish growth years of 1973-1979 this appears to have occurred; productivity change adjusted for this effect increases to over $40 \%$ of the observed productivity change 1949-1979, and to over 608 of that observed from 1965 to 1973.

In summary, empirical decomposition of the productivity residual to isolate the technical progress effects independently of subequilibrium, anticipatory behavior, and economies of scale yields important overall conclusions. These include: (i) the anomaly of the observed post-1973 productivity growth slowdown measured using conventional methods appears overstated; (ii) the primary impact generating this measured result is, 
in a one quasi-fixed input model, that of anticipatory expectations; however, if the model incorporates two quasi-fixed inputs then the primary cause is that of subequilibrium, and (iii) the productivity growth slowdown appears to have begun earlier than 1973, perhaps as early as 1965.

This empirical exercise indicates that distinct effects of various components of observed productivity growth can be identified using the procedures developed in this paper, and illustrates the richness of the decomposition framework. Significantly, the adjustments do not necessarily decrease the productivity residual or "measure of our ignorance." In fact, overall they increase the residual in all periods discussed. Nonetheless, they do indicate that much of the relative productivity growth slowdown can be attributed to a combination of these impacts. 
IV. CONCLUDING REMARKS

In these concluding remarks I briefly summarize how different existing approaches to subequilibrium productivity measurement can be viewed as special cases of the model developed here.

In many previous studies researchers have attempted to purge cyclical effects from their productivity calculations by comparing only "peak" years in the cycle. The justification offered for such a procedure is that CU rates are at their maximum so that variations from this level are not a factor affecting productivity growth. The theoretical model outlined in this paper suggests that this approach is valid provided measured $C U$ is the same in each of these peak years.

The framework presented here also reveals that $\mathrm{CU}$, calculated appropriately, involves a total output or total cost measure and thus adjusts all input shares in the productivity calculation rather than only one input, say capital. However, if utilization only of capital is varying, this approach justifies the use of a capital utilization measure to adjust the capital share in productivity calculations, as long as the shares are re-scaled to equal unity.. For example, the method advocated by Berndt and Fuss (1981) is a special case of the capacity utilization approach presented here, where the shadow value of capital-represented by Tobin's $q$--is interpreted as a measure of the utilization of capital compared to its long run equilibrium. Similarly, Hulten (1983) presents a method of adjusting productivity measures for capital utilization by recognizing shifts in cost curves from the short run revaluation of capital.

More generally, the cost CU measure with one quasi-fixed input not 
only includes all the information contained in Tobin's q--for both indicators depend on a comparison between $z_{k}$ and $p_{k}$--but also is directly dual to the quantity side. The primal output CU measure, incorporating the effects of utilization of the quasi-fixed input(s) on output, also depends on the deviation between $z_{k}$ and $p_{k}$. The shadow revaluation concept and the more conventional quantity-based capacity utilization adjustment can thus be recognized and reconciled as "two sides of the same coin."

In addition, with only one quasi-fixed input, capital, cost or output CU measures are a function only of capital utilization. In the more general case with multiple quasi-fixed inputs, the economic derivation of $\mathrm{CU}$ measures provides a justification for optimally weighting the utilization of the individual quasi-fixed inputs into an overall CU subequilibrium measure, and therefore contains more information than Tobin's $\mathrm{q}$.

The incorporation of nonstatic expectations into the models is also important. The existence of nonstatic expectations implies that if firms expect increases in output demand or input prices that will increase present value costs of investing in the future relative to the present, current anticipatory investment will be carried out which will appear excessively high given current levels of capacity. This will result in lower levels of $\mathrm{CU}$ and lower levels of productivity for variable inputs than if the firm were myopic.

Empirically this framework provides a useful structure within which to assess the productivity residual by identifying independently the effects that should not be attributed solely to technical progress. Although this study does not provide an explanation of the residual in the 
sense that the residual after the adjustments is larger than before, it does provide important insights. Analysis of the productivity residual suggests, for example, that even with CU adjustments the productivity slowdown commenced at an earlier date than is often assumed. Since dating the "productivity slowdown" and the impact of $\mathrm{CU}$ adjustments on this dating is a point of contention in the literature, the framework presented here is particularly relevant for assessing the alternative arguments.

In summary, the integration of the various technical and behavioral impacts considered in the model developed here provides a systematic framework that incorporates a number of existing approaches as special cases. It therefore provides a useful framework within which to assess, both theoretically and empirically, productivity growth and its interrelation with cyclical indicators such as Tobin's $q$ and CU. Finally, it is a particularly useful structure within which to pursue more extensive empirical research on comparison of differential international productivity trends and analysis of more micro data such as that for electric utilities, where the interactions between productivity and $\mathrm{CU}$ and the effects of fixity of different inputs are of crucial importance. 


\section{FOOTNOTES}

1 See Morrison (1984) for a related discussion of the use of this shadow value of investment. Note that the use of the gross value of capital $Z_{k} K=-G_{k} K$ or the net value $Z_{k} K=-\left(G_{k} K=G_{k} \dot{K}\right)$ depends on the relevant problem and interpretation. The net value should be used when the dynamic optimization framework is explicitly characterized, but in principle adjustment costs are not observed and therefore may not be picked up in conventional measures. This provides one reason firms may appear to be further from optimal $Y$ than is actually the case; Morrison (1984) shows that the gross measures more closely pick up trends in the traditional measures. Numerically, however, for the output and cost-CU indices the results differ very little between the net and gross specifications.

2 See Berndt, Fuss and Waverman (1979) for an extensive discussion of these types of elasticities.

3 The form of this representation for $\varepsilon_{\mathrm{Cy}}$ was developed in Morrison (1983c) from Lau (1978) with only one quasi-fixed input and no dynamic behavior. This results in $\mathrm{d} \ln \mathrm{C} / \mathrm{d} \ln \mathrm{Y}=\partial \mathrm{C} / \partial \mathrm{Y}(\mathrm{Y} / \mathrm{C})+\partial \mathrm{C} / \partial \mathrm{K}(\mathrm{dK} / \mathrm{dY})(\mathrm{Y} / \mathrm{C})=1$, since with CRTS $d \operatorname{lnK} / d \ln Y=1$. Thus $\varepsilon_{\mathrm{CY}}+\varepsilon_{\mathrm{CK}}=1$ with CRTS. The generalization is consistent with Lau's analysis.

4 Although it is not as obvious, (5) represents a conventional $\varepsilon_{\text {ct }}$ measure even without long run equilibrium imposed. To see this rewrite (5) as:

$$
\begin{aligned}
\varepsilon_{c t}=\frac{\dot{y}}{y}-\Sigma_{j} \frac{p_{j} x_{j}}{C} \frac{\dot{x}_{j}}{x_{j}}-\Sigma_{i} \frac{w_{i} v_{i}}{C} \frac{\dot{v}_{i}}{v_{i}} & +\Sigma_{j} \varepsilon_{c j}\left[\frac{\dot{x}_{j}}{x_{j}}-\frac{\dot{Y}}{Y}\right] \\
& +\sum_{j} \varepsilon_{c j}\left[\frac{\ddot{x}_{j}}{\dot{x}_{j}}-\frac{\dot{y}}{Y}\right] .
\end{aligned}
$$

The first term on the right hand side of $(5 a)$ is the traditional characterization of long run productivity. The latter two terms capture the adjustment of the "augmented" quasi-fixed input stocks to the "desired" level corresponding to exogenous variables including the new output level and includes the costs of this adjustment represented by $\ddot{x}_{j} / \dot{x}_{j}$. Because of CRTS it must be the case both that $\dot{x}_{j} / x_{j}-\dot{Y} / Y=d l n$ $\mathrm{x}_{j}-\mathrm{d} \ln \mathrm{Y}=0$ and $\ddot{\mathrm{X}}_{j} / \dot{\mathrm{x}}_{j}-\dot{\mathrm{Y}} / \mathrm{Y}=\mathrm{d} \ln \dot{\mathrm{X}}_{j}-\mathrm{d} \ln \mathrm{Y}=0$ for all $j$.

5 For further discussion of net versus gross shadow values and their empirical content, see Morrison (1984).

6 The ideas that this measure is based on are developed further in Morrison (1983d) for one quasi-fixed input, capital (K).

7 See Morrison (1983c). 
8 The basis for this assertion stems from Morrison (1983b) and Nickell (1978), where it is shown that the path of capital, or the investment plan given a current capital stock, can be represented by a flexible accelerator adjustment path toward the current "target" capital stock defined in terms of current exogenous variables plus a term capturing the effect of all future exogenous variable changes.

$$
\frac{1}{\lambda G_{\dot{K} \dot{K}}} \int_{t}^{\infty} e^{(\lambda-r)(s-t)}\left(\left(G_{K K}+r G_{\dot{K} K}\right)\left(K_{s}^{*}-K_{t}^{\star}\right)+G_{\dot{K} P} \dot{P}_{s}+G_{\dot{K} Y} \dot{Y}_{s}\right) d s \quad,
$$

where $\lambda$ is a diagonal adjustment matrix dependent on $r$ and the parameters of the variable cost function $G$. Variable input demands in turn depend on current variables including the current levels of investment in quasi-fixed factors.

10 The data on prices and quantities of output, capital, nonproduction and production labor, energy and intermediate material inputs for U.S. Manufacturing 1947-1981 were graciously provided by Ernst R. Berndt and David 0 . Wood. For a discussion of these data, which were constructed similarly to those reported in Berndt and Wood (1975), see Berndt and Wood (1984).

11 Note that any model which allows these elasticities to be calculated may be used as the basis for empirical application of this decomposition procedure. For example, any econometric model based on a short run or "restricted" cost function allowing for NCRTS can be used to determine the impact of subequilibrium and NCRTS on productivity. Distinguishing the effects of adjustment costs and nonstatic expectations, however, requires a full dynamic model and an explicit expectations process respectively. 


\section{BIBLIOGRAPHY}

Baily, Martin N. (1981), "The Productivity Growth Slowdown and Capital Accumulation," American Economic Review, Papers and Proceedings, May 1981, pp. 326-331.

Berndt, E.R., Fuss, M.A., and Waverman, L. (1979), "Empirical Analysis of Dynamic Adjustment Models of the Demand for Energy in U.S. Manufacturing Industries, 1947-1974," Final Report, Electric Power Research Institute, Palo Alto, California.

Berndt, Ernst R, and Melvin A. Fuss (1981), "Productivity Measurement Using Capital Asset Valuation to Adjust for Variations in Utilization," Institute for Policy Analysis, University of Toronto, Working Paper No. 8125, September.

Berndt, Ernst R., and David o. Wood (1975), "Technology, Prices, and the Derived Demand for Energy," Review of Economics and Statistics, 57 , No. 3, August, pp. 59-68.

Berndt, Ernst R., and David O. Wood (1984), "Energy Price Changes and the Induced Revaluation of Durable Capital in U.S. Manufacturing During the OPEC Decade," Manuscript, MIT Center for Energy Policy Research, January.

Hulten, Charles (1983), "Short Run and Long Run Cost Functions and the Measurement of Efficiency Change," Manuscript, July.

Jorgenson, Dale W., and Zvi Griliches (1967), "The Explanation of Productivity Change," Review of Economic Studies, Vol. $34(3)$, No. 99, July, pp. 249-282.

Lau, Lawrence J. (1978), "Applications of Profit Functions," in Production Economics: A Dual Approach to Theory and Applications, M.A. Fuss and D. McFadden, eds., North-Holland.

Morrison, Catherine J., (1983a), "Dynamic Factor Demands, Market Power, and the Shape of the Adjustment Cost Function," Manuscript, January.

Morrison, Catherine J., (1983b), "Structural Models of Dynamic Factor Demands with Nonstatic Expectations: An Empirical Comparison of Rational and Adaptive Expectations," Manuscript, January.

Morrison, Catherine J., (1983C), "Primal and Dual CU: An Application to Productivity Measurement in the U.S. Automobile Industry," Forthcoming, Proceedings of the 1983 Annual Conference on Current Issues in Productivity, Rutgers University, December. 
Morrison, Catherine J., (1984), "On the Economic Interpretation and Measurement of Optimal Capacity Utilization With Anticipatory Expectations," Manuscript, January.

Nickel1, S.J., (1978), The Investment Decisions of Firms, Cambridge University Press, Oxford.

Ohta, M. (1975), "A Note on the Duality Between Production and Cost Functions: Rate of Returns to Scale and Rate of Technical Progress," Economic Studies Quarterly, 25, pp. 63-65.

Solow, Robert M., (1957), "Technical Change and the Aggregate Production Function," Review of Economics and Statistics, Vol. 39, No. 5, August, pp. 312-320. 